\section{Diagnostik der idiopathischen Lungenfibrose}

Nach neueren epidemiologischen Untersuchungen gibt es für die idiopathische Lungenfibrose (idiopathische pulmonale Fibrose, IPF) einen steigenden Trend hinsichtlich Inzidenz, Prävalenz und Mortalität [1]. Da die IPF überwiegend eine Erkrankung des höheren Lebensalters ist $(2 / 3$ der Patienten sind älter als 60 Jahre), könnte dies zumindest teilweise durch die höhere allgemeine Lebenserwartung der Bevölkerung in den industrialisierten Ländern bedingt sein [2]. Die steigende Erkrankungshäufigkeit muss intensivierte Bemühungen um eine adäquate Diagnostik zur Folge haben. Im Gegensatz zum früher oft undifferenzierten Gebrauch des Begriffs „idiopathische Lungenfibrose“ als Sammeltopf für ätiologisch ungeklärte interstitielle Lungenerkrankungen sollte diese Diagnose heute nur noch für die von Katzenstein und Myers [3] definierte histologische Entität der „usual interstitial pneumonia“ (UIP) eingesetzt werden [4].

Für die Diagnostik der IPF/UIP lassen sich drei Hauptziele definieren:

1. Die differenzialdiagnostische Abgrenzung der IPF von anderen Formen der Lungenfibrose.

2. Die Objektivierung des Schweregrades der Lungenerkrankung.

3. Die Beurteilung der Prognose und eventuell erfolgversprechender Therapiemaßnahmen.

Zur Erreichung dieser Ziele stehen die folgenden diagnostischen Verfahren zur Verfügung:

1. Anamnese und klinische Untersuchung

2. Labordiagnostik

3. Lungenfunktionstests

4. radiologische Bildgebung inkl. hoch auflösender Computertomographie (HRCT)

5. Bronchoskopie mit bronchoalveolärer Lavage (BAL) und transbronchialer Biopsie (TBB)

6. Thorakoskopische, videoassistierte (VATS) oder offene Lungenbiopsie (OLB)

Im Folgenden sollen der jeweilige Beitrag der verschiedenen Methoden zur Erreichung der gesetzten Ziele kritisch hinterfragt und ein diagnostisches Konzept abgeleitet werden.

Pneumologie 2001; 55: 144-148

(C) Georg Thieme Verlag Stuttgart · New York ISSN 0934-8387
J. Behr

Schwerpunkt Pneumologie, Med. Klinik I, Klinikum Großhadern der Universität München

\section{Anamnese und klinischer Befund}

Unbestreitbar ist die oft wegweisende Bedeutung der Anamnese, mit der insbesondere die Exposition gegenüber inhalativen Noxen (anorganische und organische Stäube) am Arbeitsplatz oder während der Freizeit (z.B. Taubenzüchter etc.), die Einnahme von potenziell lungentoxischen Medikamenten sowie der Raucherstatus erfasst werden können. Auch Komplikationen wie rezidivierende Pneumothorazes bei Frauen (Lymphangioleiomyomatose, LAM) oder bei Rauchern (Histiozytosis X, HX) können wichtige Hinweise liefern. Handelt es sich um eine IPF/UIP, so sind das männliche Geschlecht, ein höheres Lebensalter, ein hoher Dyspnoeschweregrad über eine längere Zeit ( $>1$ Jahr) und ein hoher kumulativer Zigarettenkonsum mit einer raschen Progression und letztlich ungünstigeren Prognose assoziiert [5-9].

Der klinische Untersuchungsbefund ist meist unspezifisch, wenngleich inspiratorisches Knisterrasseln sowie die Ausbildung von Trommelschlegelfingern und Uhrglasnägeln gehäuft bei der IPF/UIP auftreten. Wichtig ist das Erkennen von Hinweisen auf zugrunde liegende Systemerkrankungen wie der rheumatoiden Arthritis, der Sklerodermie, des CRESTSyndroms, des M. Bechterew oder der Neurofibromatose Recklinghausen.

\section{Labor}

Die Labordiagnostik dient in erster Linie dem Nachweis respektive dem Ausschluss einer Systemerkrankung oder einer pulmonalen Vaskulitis. Das Screening umfasst die Bestimmung des Rheumafaktors, der antinukleären und antimitochondrialen Antikörper einschließlich deren Differenzierung (z.B. Jo-1-Antikörper), der antizytoplasmatischen Antikörper und der Komplementfaktoren C3 und C4. Aber auch die quantitative Messung der Immunglobuline IgG, IgA, IgM und IgE (Antikörpermangelsyndrom? Hyper-IgE-Syndrom?) und das Differenzialblutbild (Eosinophilie?) sind von Bedeutung. Bei gehäuften bakteriellen oder fungalen Infektionen muss auch die Durchführung von Leukozytenfunktionstests erwogen werden (Phygozytosedefekt? NADPH-Oxidase Defekt? Septische Granulomatose?).

\section{Lungenfunktion}

Die Lungenfunktionsprüfung ist für die Beurteilung und Objektivierung des Schweregrades der Erkrankung von großer Bedeutung. Sie sollte neben einer Spirometrie und Ganzkörperplethysmographie insbesondere den pulmonalen Gas- 
austausch mittels Diffusionsmessung (bevorzugt im „singlebreath"-Verfahren) und Blutgasanalyse in Ruhe und unter Belastung erfassen. In Frühstadien der Erkrankung können die Funktionswerte im Normbereich liegen. In der Regel findet sich aber ein restriktives Lungenfunktionsmuster (VK, TLK und RV vermindert) sowie eine Einschränkung des pulmonalen Gasaustausches in Ruhe oder zumindest unter Belastung (DLco, $\mathrm{PaO}_{2}, \mathrm{AaDO}_{2}$ ). Bei zusätzlicher bestehender, höhergradiger Bronchialobstruktion sollten differenzialdiagnostische Überlegungen in Richtung auf LAM oder HX angestellt werden. Die Spiroergometrie liefert wichtige Zusatzinformationen insbesondere durch Bestimmung der maximalen Sauerstoffaufnahme und der alveoloarteriellen Sauerstoffpartialdruckdifferenz, die auch als Verlaufsparameter dienen können [10]. Lungenfunktionsparameter sind nur in begrenztem Umfang mit entsprechenden histomorphologischen oder computertomographischen Veränderungen korreliert [11-13]. Eine stärkere Einschränkung verschiedener Lungenfunktionsparameter ist jedoch mit einer ungünstigen Gesamtprognose assoziiert $[5-7,9,10,14]$.

\section{Radiologie}

Seitens der radiologischen Bildgebung ist das HRCT in 1-mmSchichtdicke der Röntgen-Thoraxaufnahme bei weitem überlegen $[15,16]$. Sie stellt heute den Goldstandard der radiologischen Diagnostik dar, mit der Sensivitität für Lungengerüsterkrankungen von ca. 90\% [16-18]. Allerdings kann die Diagnose einer UIP mittels HRCT nur in etwa $2 / 3$ der histologisch gesicherten Fälle zuverlässig gestellt werden [19].

Hinsichtlich der interstitiellen Lungenveränderungen lassen sich grundsätzlich zwei Muster unterscheiden: 1. retikuläre Zeichnungsvermehrung mit verdickten Interlobularsepten und 2. milchglasartige Trübung des Parenchyms. Typisch für eine UIP sind fleckige, retikuläre Zeichnungsvermehrungen mit Betonung der basalen und subpleuralen Areale, die Ausprägung des retikulären Fibrosemusters ist dabei mit einer ungünstigen Prognose verknüpft [12]. Honigwabenbildung und Traktionsbronchiektasen sind in fortgeschrittenen Stadien häufig. Im Gegensatz zum retikulären ist das Milchglasmuster ein vieldeutiges Zeichen, welches sowohl auf einer intraalveolären Entzündung (Alveolitis), als auch auf einer intraalveolären Granulationsgewebsbildung oder auf einer fibrotischen Alveolarwandverdickung beruhen kann [20-22]. Nach der bisherigen Datenlage ist lediglich das retikuläre Muster mit Verdickung der interlobularen Septen als eindeutiges Zeichen einer Fibrosierung anzusehen, wobei das gleichzeitige Vorliegen eines geringen Anteils von Milchglasmuster $(<30 \%)$ für die Diagnose einer UIP akzeptabel ist, möglicherweise sogar ein günstigeres Therapieansprechen erhoffen lässt [23]. Liegt überwiegend eine milchglasartige Trübung des Lungenparenchyms vor, sind unterschiedliche pathomorphologische Korrelate denkbar (DIP/RBILD, NSIP, BOOP, exogenallergische Alveolitis etc.), so dass besonders in diesen Fällen eine histologische Diagnosesicherung angestrebt werden sollte. Ähnliches gilt für flächenhafte oder strangförmige intrapulmonale fibrotische Areale.

\section{Bronchoskopie, bronchoalveoläre Lavage (BAL) und transbronchiale Biopsie (TBB)}

Die Bronchoskopie mit BAL und TBB trägt nur in begrenztem Umfang zur Abklärung einer Lungengerüsterkrankung bei. Wenn die Diagnosen Sarkoidose, Infektion, Alveolarzellkarzinom oder eosinophile Pneumonie infrage kommen, lassen sich mit diesen Methoden häufig wegweisende Befunde erheben. Die definitive Diagnosestellung einer idiopathischen Lungenfibrose ist dagegen mit diesen Verfahren generell nicht möglich. Eine Lymphozytose ( $>50 \%$ ) oder eine Eosinophilie $(>25 \%)$ sollten jedoch Zweifel an der Diagnose einer IPF/UIP wecken. Auch prognostische Aussagen auf der Basis der BALAnalyse sind nur begrenzt möglich. Es gibt Hinweise darauf, dass eine Lymphozytose mit einer eher günstigen Prognose und besserem Ansprechen auf Kortikosteroide assoziiert ist, während eine isolierte neutrophile Granulozytose, insbesondere in Verbindung mit einer Eosinophilie eher auf ein progressives Krankheitsgeschehen hinweist [5,24-26].

Die bronchoskopische Diagnostik mittels BAL ist nicht völlig risikolos, insbesondere bei älteren Patienten mit Lungenfibrose wurde in Einzelfällen eine Aktivierung des Krankheitsprozesses nach BAL, zum Teil mit letalem Ausgang beobachtet [27]. Die Indikationsstellung muss daher auch hier kritisch erfolgen, insbesondere unter dem Aspekt einer möglichen therapeutischen Konsequenz.

\section{Histologie}

Die Lungenfibrosen unbekannter Ätiologie wurden von Liebow nach histologischen Kriterien in 5 Gruppen eingeteilt [28]. Diese histologische Klassifikation hat allerdings klinisch relevanten Fragestellungen nach Therapie und Prognose nur in geringem Umfang Rechnung getragen. Die oft nur geringe klinische Relevanz des histologischen Befundes einer Lungenbiopsie führte konsequenterweise zu einem seltenen Einsatz dieses diagnostischen Verfahrens durch die Kliniker. Nach einer Untersuchung von Johnston et al. aus dem Jahr 1997 wurde nur bei $12 \%$ der Patienten mit Lungenfibrose eine offene oder thorakoskopische Lungenbiopsie durchgeführt [2]. Die allgemeine Unsicherheit im Umgang mit diesen Patienten spiegelt sich auch in der Tatsache wider, dass nur bei $52 \%$ der Patienten mit IPF eine spezifische (immunsuppressive) Therapie eingeleitet wurde [2].

1998 haben Katzenstein und Myers die histologische Klassifikation der Lungenfibrosen unbekannter Ätiologie überarbeitet und sind $\mathrm{zu}$ einer neuen Klassifikation gelangt, die den klinischen Erfordernissen weit mehr entgegenkommt [3]. Tab. 1 zeigt eine Gegenüberstellung der alten (Liebow) und neuen (Katzenstein) Klassifikation. Eine wesentliche Neuerung hierbei ist die Einführung der „nonspecific interstitial pneumonia“ (NSIP) als histologisch von der UIP abgrenzbare Erkrankung mit gutem Ansprechen auf Glukokortikosteroide und klinisch weitaus günstigerem Verlauf $[3,29,30]$. Obwohl man darüber streiten kann, ob der Begriff „NSIP“ glücklich gewählt ist, muss man anerkennen, dass die Stellung dieser Diagnose, die bei ca. $20 \%$ der Patienten mit interstitiellen Pneumonien vorliegt, eine entscheidende Bedeutung für das klinische Management der Patienten hat. 
Tab. 1 Pathologische Klassifikation der interstitiellen Pneumonien

\begin{tabular}{|c|c|}
\hline Liebow et al. 1969 [28] & Katzenstein et al. 1998 [3] \\
\hline $\begin{array}{l}\text { Usual Interstitial Pneumonia } \\
\text { (UIP) }\end{array}$ & $\begin{aligned} \text { - Usual Interstitial Pneumonia (UIP) } \\
\text { - Nonspecific Interstitial Pneumonia } \\
\text { (NSIP) } \\
\text { - Acute Interstitial Pneumonia (AIP) }\end{aligned}$ \\
\hline $\begin{array}{l}\text { Desquamative Interstitial } \\
\text { Pneumonia (DIP) }\end{array}$ & $\begin{array}{l}\text { DIP/Respiratory Bronchiolitis Inter- } \\
\text { stitial Pneumonia (DIP/RBILD) }\end{array}$ \\
\hline $\begin{array}{l}\text { Bronchiolitis Obliterans } \\
\text { Interstitial Pneumonia (BIP) }\end{array}$ & $\begin{array}{l}\text { Bronchiolitis Obliterans Organizing } \\
\text { Pneumonia (BOOP) }\end{array}$ \\
\hline $\begin{array}{l}\text { Lymphoid Interstitial Pneumo- } \\
\text { nia (LIP) }\end{array}$ & $\begin{array}{l}\text { nicht enthalten (heute unter die } \\
\text { lymphoprolifertiven Erkrankungen } \\
\text { subsumiert) }\end{array}$ \\
\hline $\begin{array}{l}\text { Giant Cell Interstitial Pneumo- } \\
\text { nia (GIP) }\end{array}$ & $\begin{array}{l}\text { nicht enthalten (heute: Hartmetall- } \\
\text { fibrose) }\end{array}$ \\
\hline
\end{tabular}

Die Gewinnung einer thorakoskopischen (VATS) oder offenen Lungenbiopsie (OLB), möglichst aus einem nach HRCT-Kriterien charakteristisch veränderten Lungenareal ist der Goldstandard in der Diagnostik der idiopathischen Lungenfibrose. Sie allein erlaubt eine weitgehend sichere Abgrenzung der verschiedenen von Katzenstein charakterisierten Formen der Lungenfibrose. Allerdings gibt es - wenn auch selten $(<10 \%)$ - nicht diagnostische, unspezifische und sogar normale Lungenbiopsiebefunde bei UIP Patienten. Nach einer neueren Untersuchung ist die Lungenbiopsie (OLB oder VATS) bei Fibrosepatienten ohne relevanten Funktionsverlust und mit relativ geringem Risiko möglich, wobei die Studienpopulation insgesamt nur mittelgradige Funktionseinschränkungen aufwies [31]. Allerdings hatte auch eine Subgruppenanalyse der schwerer erkrankten Patienten (FVC $<60 \%$ ) in dieser Untersuchung keine erhöhte Morbidität oder Mortalität ergeben [31].

Problematisch bleibt, dass die therapeutische Konsequenz im Allgemeinen relativ uniform ist, da die Mehrzahl der Patienten zumindest probatorisch Glukokortikosteroide, zum Teil in Kombination mit Azathioprin oder Cyclophosphamid erhalten [32]. Dennoch erscheint es im Einzelfall durchaus relevant die histologische Zuordnung eines Patienten zu kennen, da sich zumindest die Dauer und Intensität eines Behandlungsversuchs mit Immunsuppressiva begrenzen lassen, wenn eine UIP gesichert ist, während bei Nachweis einer NSIP eine intensive und langfristige (evtl. sogar lebenslange) Kortikosteroidtherapie notwendig ist. Die Indikation zur Lungenbiopsie muss daher individuell vor dem Hintergrund der aktuellen Funktionseinschränkung, des Alters und möglicher therapeutischer Konsequenzen gestellt werden.

\section{Diagnostisches Konzept}

Grundsätzlich beruht die Diagnose einer IPF/UIP immer auf einer vollständigen Evaluation des Patienten, auch die Lungenbiopsie erlaubt die Diagnosestellung nur im Kontext mit den klinischen, funktionsanalytischen und radiologischen Befunden. Allerdings ist die Möglichkeit eine IPF/UIP auch in Abwesenheit einer chirurgischen Lungenbiopsie zu diagnostizieren in den ATS/ERS Empfehlungen enthalten [4] und soll nach den in Tab. 2 dargestellten Haupt- und Nebenkriterien
Tab. 2 Diagnosekriterien der IPF/UIP in Abwesenheit einer chirurgischen Lungenbiopsie (nach [4])

Alle 4 Hauptkriterien und 3 von 4 Nebenkriterien müssen erfüllt sein. Hauptkriterien

1. Ausschluss bekannter Ursachen einer interstitiellen Lungenerkrankung inkl. der Systemerkrankungen

2. restriktives Lungenfunktionsmuster und Gasaustauschstörung

3. beidseits basale retikuläre Verdichtungen subpleural im HRCT, Milchglastrübung $<30 \%$

4. BAL oder TBB ohne Hinweise auf eine alternative Diagnose

Nebenkriterien

1. Alter $>50$ Jahre

2. langsam zunehmende Belastungsdyspnoe ohne andere Ursache

3. Erkrankungsdauer $>3$ Monate

4. inspiratorisches Knisterrasseln beidseits basal

erfolgen. Bei kritischer Würdigung des zur Verfügung stehenden therapeutischen Repertoires und der bestehenden internationalen Empfehlungen lässt sich für die klinische Praxis folgendes Procedere empfehlen:

1. Die Durchführung der nichtinvasiven Diagnostik (Anamnese, klinischer Befund, Labordiagnostik, Lungenfunktionsprüfung und HRCT) ist zur Eingrenzung des vorliegenden Krankheitsbildes obligat und dient der Stellung einer Arbeitsdiagnose (IPF/UIP oder alternative Diagnose?).

2. Entscheidung über die Durchführung einer chirurgischen Lungenbiopsie, die immer dann obligat sein sollte, wenn der Patient unter 60 Jahre alt ist, keine zusätzlichen relevanten Organstörungen vorliegen und klinische, funktionsanalytische oder HRCT-Befunde nicht typisch für eine UIP sind. Auch bei typischer Befundkonstellation für eine IPF/UIP ist in dieser Patientengruppe die histologische Klärung wegen der prognostischen Aussagekraft und als Grundlage für den langfristigen Einsatz immunsuppressiv wirkender Medikamente empfehlenswert. Bei der Entscheidung für eine Lungenbiopsie bringt die bronchoskopische Diagnostik in der Regel keine relevante Zusatzinformation und kann daher entfallen.

3. Einer chirurgischen Lungenbiopsie sollten auch Patienten mit Verdacht auf eine pulmonale Vaskulitis zugeführt werden, wenn nicht extrapulmonale Organbeteiligungen eine einfachere Diagnosesicherung erlauben (z.B. Hautbiopsie bei Churg-Strauss-Syndrom).

4. Bei Hinweisen auf eine Kollagenose sollte diese nach den jeweiligen Diagnosekriterien erhärtet werden. Im Fall einer Systemerkrankung, z.B. der Sklerodermie, kann die BAL therapierelevante Zusatzinformationen liefern [33], und sollte erfolgen, wenn nicht bereits wegen der Grunderkrankung per se eine immunsuppressive Therapie eingeleitet werden muss.

5. Bei älteren Patienten (>60 Jahre) mit typischen Befunden in der nichtinvasiven Diagnostik und dem HRCT-Bild der IPF/UIP sollte vor Beginn einer immunsuppressiven Therapie eine Bronchoskopie mit BAL und TBB zum Ausschluss einer Infektion oder eines Alveolarzellkarzinoms erfolgen. Ergeben sich auch hier keine Befunde, die gegen eine IPF/ UIP sprechen, ist die Lungenbiopsie nicht obligat.

6. Bei älteren Patienten (> 60 Jahre), die nach Ausschöpfung der nichtinvasiven und der bronchoskopischen Diagnostik 
keine eindeutige Befundkonstellation aufweisen, müssen das Risiko einer probatorischen immunsuppressiven Therapie über eine Dauer von mindestens 3-6 Monaten und das Risiko der Lungenbiopsie sowie die Wahrscheinlichkeit einer damit verbundenen, abweichenden therapeutischen Konsequenz gegeneinander abgewogen werden. Vor allem wenn schwere Lungenfunktionseinschränkungen (VC $<60 \%$, DLco $<50 \%$ ) oder zusätzliche Organfunktionsstörungen bestehen (z.B. Herzinsuffizienz), wird diese Abwägung gegen eine Lungenbiopsie ausfallen müssen.

\author{
Abkürzungen \\ $\mathrm{AaDO}_{2}=$ Alveoloarterielle Sauerstoffpartialdruckdifferenz \\ AIP = Akute interstitielle Pneumonie \\ BAL = Bronchoalveoläre Lavage \\ BIP = Bronchiolitis obliterans interstitielle Pneumonie \\ BOOP = Bronchiolitis obliterans organisierende Pneumonie \\ DLco = Diffusionskapazität der Lunge für Kohlenmonoxid \\ GIP = Interstitielle Pneumonie mit Riesenzellen \\ (Giant cells) \\ HRCT = Hoch auflösende Computertomographie \\ $\mathrm{HX}=$ Histiozytose $\mathrm{X}$ \\ IPF = Idiopathische pulmonale Fibrose \\ LAM = Lymphangioleiomyomatose \\ LIP = Lymphoide interstitielle Pneumonie \\ NSIP = Unspezifische (nonspecific) interstitielle Pneumonie \\ OLB = Offene (chirurgische) Lungenbiopsie \\ $\mathrm{PaO}_{2}=$ Arterieller Sauerstoffpartialdruck \\ RBILD = Respiratorische Bronchiolitis interstitielle Lungen- \\ erkrankung \\ $\mathrm{RV} \quad=$ Residualvolumen \\ TBB = Transbronchiale Biopsie \\ TLK = Totale Lungenkapazität \\ UIP = Gewöhnliche (usual) interstitielle Pneumonie \\ VATS = Videoassistierte thorakoskopische Lungenbiopsie \\ VK = Vitalkapazität
}

\section{Literatur}

${ }^{1}$ Mannino DM, Etzel RA, Parrish RG. Pulmonary fibrosis deaths in the United States, 1979-1991: an analysis of multiple-cause mortality data. Am J Respir Crit Care Med 1996; 153: 1548 1552

${ }^{2}$ Johnston IDA, Prescott RJ, Chalmers JC, Rudd RM for the Fibrosing Alveolitis Subcommittee of the Research Committee of the British Thoracic Society. British Thoracic Society study of cryptogenic fibrosing alveolitis: current presentation and initial management. Thorax 1997; 52: 38 - 44

${ }^{3}$ Katzenstein ALA, Myers JL. Idiopathic pulmonary fibrosis. Clinical relevance of pathologic classification. Am J Respir Crit Care Med 1998; 157: $1301-1315$

${ }^{4}$ Joint Statement of the American Thoracic Society (ATS) and European Respiratory Society (ERS). Idiopathic pulmonary fibrosis: diagnosis and treatment. International consensus statement. Am J Respir Crit Care Med 2000; 161: 646-664

${ }^{5}$ Rudd RM, Haslam PL, Turner-Warwick M. Cryptogenic fibrosing alveolitis relationships of pulmonary physiology and bronchoalveolar lavage to treatment and prognosis. Am Rev Respir Dis $1981 ; 124: 1-8$

${ }^{6}$ Turner-Warwick M, Burrows P, Johnson A. Cryptogenic fibrosing alveolitis: clinical features and their influence on survival. Thorax 1980; 35: $171-180$
${ }^{7}$ Schwartz DA, Helmers RA, Galvin JR, Van Fossen DS, Frees KL, Dayton CS, Burmeister LF, Hunninghake GW. Determinants of survival in idiopathic pulmonary fibrosis. Am J Respir Crit Care Med 1994; 149: 450-454

${ }^{8}$ Tukiainen P, Taskinen E, Holsti P, Korhola O, Valle M. Prognosis of cryptogenic fibrosing alveolitis. Thorax 1983; 38: 349-355

${ }^{9}$ Hiwatari N, Shimura S, Sasaki T, Aikawa T, Ando Y, Ishihara H, Sekizawa K, Sasaki H, Takishima T. Prognosis of idiopathic pulmonary fibrosis in patients with mucous hypersecretion. Am Rev Respir Dis 1991; 143: 182 - 185

${ }^{10}$ Fulmer JD, Roberts WC, van Gal ER, Crystal RG. Morphologicphysiologic correlates of the severity of fibrosis and degree of cellularity in idiopathic pulmonary fibrosis. J Clin Invest 1979; 63: $665-676$

${ }^{11}$ Keogh BA, Crystal RG. Clinical significance of pulmonary function tests. Chest 1980; 78: 856-865

12 Gay SE, Kazerooni EA, Toews GB, Lynch III J, Gross BH, Cascade PN, Spizarny DL, Flint A, Schorck MA, Whyte RI, Popovich J, Hyzy $\mathrm{R}$, Martinez FJ. Idiopathic pulmonary fibrosis: predicting response to therapy and survival. Am J Respir Crit Care Med 1998; 157: $1063-1072$

${ }^{13}$ Xaubet A, Agusti C, Luburich P, Rova J, Monton C, Ayuso MC, Barbera JA, Rodriguez-Roisin R. Pulmonary function tests and CT scan in the management of idiopathic pulmonary fibrosis. Am J Respir Crit Care Med 1998; 158: 431 - 436

${ }^{14}$ Erbes R, Schaberg T, Loddenkemper R. Lung function tests in patients with idiopathic pulmonary fibrosis: are they helpful for predicting outcome?. Chest 1997; 111: $51-57$

${ }^{15}$ Orens JB, Kazerooni EA, Martinez FJ, Curtis JL, Gross BH, Flint A, Lynch III JP. The sensitivity of high-resolution CT in detecting idiopathic pulmonary fibrosis proved by open lung biopsy: a prospective study. Chest 1995; 108: 109-115

${ }^{16}$ Grenier P, Valeyre D, Cluzel P, Brauner MW, Lenoir S, Chastand C. Chronic diffuse interstitial lung disease: diagnostic value of chest radiography and high-resolution CT. Radiology 1991; 179: $123-132$

${ }^{17}$ Mathieson JR, Mayo JR, Staples CA, Muller NL. Chronic diffuse infiltrative lung disease: comparison of diagnostic accuracy of CT and chest radiography. Radiology 1989; 171: 111-116

18 Tung KT, Wells AU, Rubens MB, Kirk JM, du Bois RM, Hansell DM. Accuracy of the typical computed tomographic appearances of fibrosing alveolitis. Thorax 1993; 48: 334-338

${ }^{19}$ Swensen S, Aughenbaugh G, Myers J. Diffuse lung disease: diagnostic accuracy of CT in patients undergoing surgical biopsy of the lung. Radiology 1997; 205: 229-234

${ }^{20}$ Muller N, Staples C, Miller R, Vedal S, Thurlbeck W, Ostrow D. Disease activity in idiopathic pulmonary fibrosis: CT and pathologic correlation. Radiology 1987; 165: 731 - 734

${ }^{21}$ Remy-Jardin M, Giraud F, Remy J, Copin MC, Gosselin B, Duhamel A. Importance of ground-glass attenuation in chronic diffuse infiltrative lung disease: pathologic-CT correlation. Radiology 1993; 189: 693-698

${ }^{22}$ Nishimura K, Kitaichi M, Izumi T, Nagai S, Kanaoka M, Itoh H. Usual interstitial pneumonia: histologic correlation with highresolution CT. Radiology 1992; 182: 337-342

${ }^{23}$ Wells UA, Rubens MB, du Bois RM, Hansell DM. Serial CT in fibrosing alveolitis: prognostic significance of the initial pattern. Am J Roentgenol 1993; 161: 1159-1165

${ }^{24}$ Watters LC, Schwartz MI, Cherniack RM, Waldron JA, Dunn TL, Stanford DE, King Jr TE. Idiopathic pulmonary fibrosis: pretreatment bronchoalveolar lavage cellular constituents and their relationships with lung histopathology and clinical response to therapy. Am Rev Respir Dis 1987; 135: 696 - 704

${ }^{25}$ McCormack FX, King Jr TE, Voelker DR, Robinson PC, Mason RJ. Idiopathic pulmonary fibrosis: abnormalities in the bronchoal- 
veolar lavage content of surfactant protein A. Am Rev Respir Dis 1991; 144: 160-166

${ }^{26}$ Schwartz DA, Van Fossen DS, Davis CS, Helmers RA, Dayton CS, Burmeister LF, Hunninghake GW. Determinants of progression in idiopathic pulmonary fibrosis. Am J Respir Crit Care Med 1994; 149: $444-449$

${ }^{27}$ Hiwatari N, Shimura S, Takashima T, Shirato K. Bronchoalveolar lavage as a possible cause of acute exacerbation in idiopathic pulmonary fibrosis patients. Tohoku J Exp Med 1994; 174: 379386

${ }^{28}$ Liebow AA, Carrington CB. The interstitial pneumonias. In: Simon M, Potchen EJ, LeMay M (eds). Frontiers of pulmonary radiology, 1. New York: Grune and Stratton, 1969: 102-141

${ }^{29}$ Bjoraker JA, Ryu JH, Edwin MK, Myers JL, Tazelaar HD, Schroeder DR, Offorf KP. Prognostic significance of histopathologic subsets in idiopathic pulmonary fibrosis. Am J Respir Crit Care Med 1998; 157: 199-203

${ }^{30}$ Nagai S, Kitaichi M, Itoh H, Nishimura K, Izumi T, Colby TV. Idiopathic nonspecific interstitial pneumonia/fibrosis: comparison with idiopathic pulmonary fibrosis and BOOP. Eur Respir J 1998; 12: 1010-1019

${ }^{31}$ Daniil Z, Gilchrist FC, Marciniak SJ, Pantelidis P, Goldstraw P, Pastorino U, du Bois RM. The effect of lung biopsy on lung function in diffuse lung disease. Eur Respir J 2000; 16: 67 - 73

32 Kramer MR, Berkman N, Mintz B, Godfrey S, Saute M, Amir G. The role of open lung biopsy in the management and outcome of patients with diffuse lung disease. Ann Thorac Surg 1998; 65: $198-202$

${ }^{33}$ Behr J, Vogelmeier C, Beinert T, Meurer M, Krombach F, König G, Fruhmann G. Bronchoalveolar lavage for evaluation and management of scleroderma disease of the lung. Am J Respir Crit Care Med 1996; 154: 400-406

Priv.-Doz. Dr. med. J. Behr

Leiter des Schwerpunkts Pneumologie

Medizinische Klinik I

Klinikum Großhadern der Universität München

Marchioninistr. 15

81377 München

E-mail: jbehr@med1.med.uni-muenchen.de
BUCHBESPRECHUNG

Ficker J. H. Oszilloresistometrie beim Schlafapnoe-Syndrom. Georg Thieme, Stuttgart, 2000. 63 S., 41 Abb., kart. DM 59,-. ISBN 3-13-105621-5

Das vorliegende Buch beschreibt Grundlagen, Entwicklung, Untersuchungen zur Validität und klinische Anwendung der oszillatorischen Messung der pharyngealen Widerstände beim Schlafapnoesyndrom. Das Verfahren war ursprünglich für die Lungenfunktionsdiagnostik entwickelt worden. Eine „Störquelle“ der Messungen des Atemwegswiderstandes stellten die kollapsiblen Anteile des Pharynx dar. Genau diese Abschnitte sind aber nachts bei schlafbezogenen obstruktiven Atmungsstörungen verengt bzw. verschlossen. Daher kann mit Hilfe der Oszilloresistometrie der von CPAP-Generatoren abgegebene Druck je nach Öffnungsweite der oberen Atemwege während der Nacht automatisch titriert werden. Wesentliche Grundlagen zum klinischen Einsatz des Systems wurden vom Autor erarbeitet und experimentell geprüft. Dies betrifft die Detektion obstruktiver Ereignisse, die Differenzierung zentraler Apnoen, die Geschwindigkeit und das Ausmaß der Regulation des Beatmungsdruckes sowie den Einfluss auf Schlafparameter. Die Untersuchungen stellen einen Teil der Habilitationsschrift dar.

Das Buch ist sehr klar gegliedert und führt in anschaulicher Weise u.a. mit Hilfe vieler Originalkurven in die Methodik ein. Sensitivität, Spezifität, Fehlerquellen, Stellenwert und Anwendung sind kompetent und gut verständlich geschildert. Die Lektüre ist für alle, die mit Lungenfunktionsdiagnostik und Therapie schlafbezogener Atmungsstörungen befasst sind, sehr zu empfehlen.

H. Hein, Großhansdorf 